\title{
Hhex drives B cells down memory lane
}

\author{
The combination of single-cell RNA-seq and in vivo CRISPR-Cas9 screens reveal a new circuit that directs germinal \\ center $B$ cells toward a memory $B$ cell phenotype during viral infection.
}

\section{Kim L. Good-Jacobson and Joanna R. Groom}

C urrently, the world is collectively holding its breath for the development of an effective vaccine to SARS-CoV-2. Memory B cell (MBC) formation is a cornerstone of immune protection and essential for vaccination success. Despite their importance, the identity of MBC precursors and the transcriptional mechanisms driving $\mathrm{MBC}$ formation are incomplete. In this issue of Nature Immunology, Laidlaw et al. combine single-cell RNA-sequencing (RNA-seq) and CRISPR-Cas9 screening approaches to identify the transcription factor Hhex as an essential, time-dependent regulator of memory formation ${ }^{1}$. Within germinal center (GC) reactions, Hhex acts in concert with the corepressor Tle 3 to induce Bcl-2 and Ski, both of which could rescue MBC frequency in the absence of Hhex. This transcriptional circuit directs B cells away from ongoing participation in GC reactions and promotes memory B cell differentiation. Together, the findings of Laidlaw et. al support an instructive model in which a subset of GC B cells is actively driven toward a memory B cell phenotype by Hhex, suggesting this process has the potential to be steered therapeutically.

Humoral memory encompasses two main populations: recirculating $\mathrm{MBCs}$ and long-lived antibody-secreting plasma cells ${ }^{2}$. While a constant source of serum antibody provides a first-line of defense against a secondary infection, long-lived plasma cells are terminally differentiated and therefore cannot increase their numbers in response to a replicating pathogen. Instead, it is MBCs that hold the key to effective secondary responses. MBCs rapidly produce high-affinity antibody upon reactivation, helping to clear the infection before it can cause damage. Determining how to pull the molecular levers that drive B cell differentiation toward MBC production is critical for rational vaccine design and new treatments for MBC immunodeficiencies. Yet, this has remained a stubbornly elusive goal. Reciprocal transcriptional circuits controlled by distinct transcription factors have been described for both GC and plasma cell populations ${ }^{3,4}$. By contrast,

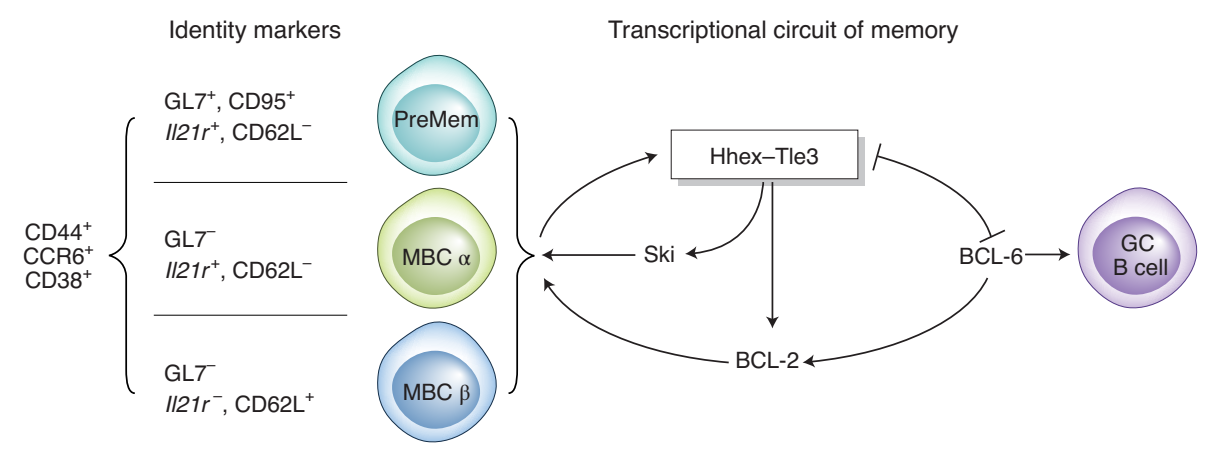

Fig. 1 | Surface marker expression and transcriptional circuit for subpopulations of memory B cells. Compilation of identity markers that indicate distinct PreMem and MBC subsets. Laidlaw et al. validated CD62L and CD44 expression at the protein level. Other markers were revealed by transcriptional analysis in the current study; transcriptional networks of Mem $\alpha$ and Mem $\beta$ cells were correlated with those of previously described CD80-CD273- and CD80 ${ }^{+} \mathrm{CD} 273^{+}$subsets, respectively ${ }^{8}$. Hhex and Tle3 physically interact in $\mathrm{MBC}$ s and serve as a transcriptional hub to promote $\mathrm{MBC}$ differentiation at the expense of GC B cells. Bcl- 6 and Hhex transcriptionally repress each other and may balance $\mathrm{GC}$ and $\mathrm{MBC}$ fates, respectively. $\mathrm{Bcl}-2$ and Ski are downregulated in the absence of Hhex, and their overexpression can compensate for the loss of Hhex to steer cells toward a memory B cell phenotype.

no unique transcription factors had been defined for the MBC population. Recent studies published by Laidlaw and colleagues and others have revealed a number of key molecules, such as IL-9R, ephrin-B1 and CCR6, which define a subset of MBC precursors within the $\mathrm{GC}^{5-7}$. Identifying MBC precursors had begun to illuminate the pathway via which B cells in the GC transition toward a MBC fate, but the underlying molecular circuits that regulate MBC differentiation remained in the dark.

In the present study, Laidlaw et al. use a single-cell RNA-seq system to identify individual B cell populations that exist at the peak of GC reactions following acute lymphocytic choriomeningitis virus (LCMV) infection ${ }^{1}$. This analysis led to the identification of eight discrete transcriptional signatures, which correlated with previously identified GC populations. The MBC cluster featured high expression of $\mathrm{Cd} 38, \mathrm{Bcl} 2$ and Zeb2 and low Bcl6 expression. This population was further dissected to reveal three subpopulations that fall along a spectrum of transcriptional identity that exists between GC and MBC signatures $^{5}$, which the authors labeled Mem $\alpha$, Mem $\beta$ and PreMem, and that appear related to previously identified MBC subsets (Fig. 1) ${ }^{8}$. Deletion of target MBC genes was assessed with an in vivo CRISPRCas9 screen, revealing Hhex and Tle 3 as novel regulators of $\mathrm{MBC}$ differentiation. Subsequently, both Hhex and Tle3 were confirmed to be sufficient to actively drive $\mathrm{MBC}$ formation, as their overexpression led to increases in memory formation, with a reciprocal decrease in GC B cells.

To determine how Hhex modulates MBC formation, Laidlaw et al. analyzed key factors induced in MBCs in its absence. The inhibitor of apoptosis $\mathrm{Bcl}-2$, a known regulator of MBCs, was reduced in Hhex-deficient cells. Use of a $B c l 2$ overexpression system restored the $\mathrm{MBC} / \mathrm{GC}$ ratio in Hhex-deficient cells, demonstrating that increased levels of Bcl-2 can compensate for the loss of Hhex during MBC development. Similarly, overexpression of the transcription factor Ski was also capable of restoring MBC differentiation in Hhex-deficient cells. Thus, Hhex appears to act as a hub for multiple 
transcriptional circuits that work together to promote MBC development.

It is not yet clear how Hhex and Tle 3 are induced to promote $\mathrm{MBC}$ formation during GC reactions and which specific intrinsic or extrinsic factors are involved. However, the authors provide intriguing clues to the regulation of Hhex within the GC. GC $B$ cells are defined by their expression of Bcl-6. Mining publicly available Bcl-6 ChIP analysis datasets indicated that Bcl- 6 and an important corepressor, Bcor, directly bind the Hhex locus. Subsequently, the authors demonstrated that Bcl- 6 overexpression leads to the downregulation of Hhex. As GC cells are reduced when Hhex is overexpressed, the authors suggest a relationship of reciprocal repression may exist between Hhex and Bcl-6. Indeed, Bcl-6 was upregulated in Hhex-deficient cells and multiple Bcl-6 transcriptional targets were also differentially regulated. While these data provide a new understanding of the precise transcriptional balance that maintains GC and MBC fates, the upstream switch that turns on Hhex and other mediators of memory formation remain elusive. For this goal, kinetic trajectory single-cell analysis may reveal the identity of the earliest memory precursor, rather than characterizing PreMem cells at the peak of their formation. Nevertheless, the identification of the Hhex-Tle 3 axis will assist further studies that investigate the ontogeny of B cell memory.

The two key characteristics of the memory B cell population critical for effective protective immunity are (1) the ability to persist in the absence of infection and (2) the rapid expansion and production of plasmablasts upon secondary infection. When memory cells lacking Hhex were transferred into naive animals to test their recall response, they exhibited diminished recall responses on a per cell basis, confirming the reduced differentiation toward memory, but they did not directly influence antiviral secondary responses. Furthermore, when Hhex was deleted following the generation of MBCs, there was no effect on the maintenance of $B$ cell memory. Together, these results suggest that the role of Hhex is confined to the generation of GC-derived memory, and it is not involved in the maintenance or function of B cell memory.

How the MBC fate decision is made in space and time is also a critical consideration. The microenvironmental cues that initially promote the transition of GC B cells toward $\mathrm{MBC}$ formation over plasma cell differentiation remain to be determined, although there is evidence to suggest that MBC fate is extrinsically determined by cell location. Previous work identified the chemokine receptor CCR6 as an early marker of PreMem cells within GCs ${ }^{6}$, which is confirmed by the current study ${ }^{1}$. Additionally, PreMem cells have previously been identified on the edge of the GC microstructure and, specifically, within the light zone ${ }^{5-7}$. These findings are consistent with the observation that the MBC gene signature more closely resembles the signatures of the light zone than those of the dark zone GC B cells. However, whether stochastic positioning of cells within the light zone and/or a specific cue provided by follicular T cells or dendritic cells is required for inducing MBC differentiation remains to be determined ${ }^{2}$. While multiple molecules that instruct cell positioning were shown to be regulated in the absence of Hhex, many of these are direct transcriptional targets of Bcl-6. As Bcl-6 has a known role in direct $\mathrm{T}$ follicular helper cell migration and positioning, its repression may instruct the position of MBCs. Thus, there is currently little understanding of how the interplay and order of cell positioning lead to transcriptional regulation of GC cell fates, and this should be actively pursued in future studies.

Bridging the divide between developing a fundamental understanding of regulatory molecular networks and being able to modulate the formation of MBCs therapeutically is a key goal to pursue. One difficulty, commonly encountered with transcription factors, is that Hhex is important in other cell types, such as lymphoid progenitors ${ }^{10}$. To circumvent this, the next step would be to use the groundwork from this study to understand both the upstream regulators and downstream targets of Hhex, which could illuminate new therapeutic targets and thus significantly advance the capability of modulating MBCs. Furthermore, fundamental insights into how MBC development is disrupted in chronic infection could be gained by examining of the MBC transcriptional networks identified by Laidlaw et al. Understanding the relationship between the microenvironment, in either a chronic or an acute resolving infection, the positioning within peripheral tissues and how these facilitate rapid local recall is also likely to yield valuable insight into the factors that underpin the longevity of protective memory ${ }^{11,12}$. Given the current COVID-19 pandemic, these concerns are at the forefront of the global challenge to define and elicit long-lived protection against emerging viral pathogens. Here, Laidlaw et al. offer a starting point, with markers and tools to identify these memory subsets and facilitate these studies.

Kim L. Good-Jacobson (D) 1,2四 and Joanna R. Groom (iD) 3,4凶

${ }^{1}$ Department of Biochemistry and Molecular Biology, Monash University, Clayton, Victoria, Australia. ${ }^{2}$ Biomedicine Discovery Institute, Monash University, Clayton, Victoria, Australia. ${ }^{3}$ Division of Immunology, Walter and Eliza Hall Institute of Medical Research, Parkville, Victoria, Australia. ${ }^{4}$ Department of Medical Biology, University of Melbourne, Parkville, Victoria, Australia.

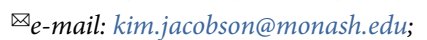
groom@wehi.edu.au

Published online: 7 August 2020 https://doi.org/10.1038/s41590-020-0763-9

\section{References}

1. Laidlaw, B. J., Lihui, D., Xi, J., Vazquez, S. E. \& Cyster, J. G.

Nat. Immunol. https://doi.org/10.1038/s41590-020-0713-6 (2020).

2. Good-Jacobson, K. L. Immunol. Rev. 284, 67-78 (2018).

3. Dent, A. L., Shaffer, A. L., Yu, X., Allman, D. \& Staudt, L. M. Science 276, 589-592 (1997).

4. Shaffer, A. L. et al. Immunity 17, 51-62 (2002).

5. Laidlaw, B. J. et al. J. Exp. Med. 214, 639-649 (2017).

6. Suan, D. et al. Immunity 47, 1142-1153.e4 (2017).

7. Takatsuka, S. et al. Nat. Immunol. 19, 1025-1034 (2018).

8. Zuccarino-Catania, G. V. et al. Nat. Immunol. 15, 631-637 (2014).

9. Hatzi, K. et al. J. Exp. Med. 212, 539-553 (2015).

10. Jackson, J. T. et al. Blood 125, 803-814 (2015).

11. Cooper, L. \& Good-Jacobson, K. L. Immunol. Cell Biol. 98 , 456-466 (2020).

12. Allie, S. R. et al. Nat. Immunol. 20, 97-108 (2019).

Competing interests

The authors declare no competing interests. 